TITLE:

\title{
Angular distribution of energy spectrum in two-dimensional $\beta$ - plane turbulence in the long-wave limit
}

\section{$\operatorname{AUTHOR}(\mathrm{S})$ :}

Saito, Izumi; Ishioka, Keiichi

\section{CITATION:}

Saito, Izumi ... [et al]. Angular distribution of energy spectrum in two-dimensional $\beta$-plane turbulence in the long-wave limit. Physics of Fluids 2013, 25(7): 076602

ISSUE DATE:

2013-07-18

URL:

http://hdl.handle.net/2433/178670

RIGHT:

(C) 2013 AIP Publishing LLC 


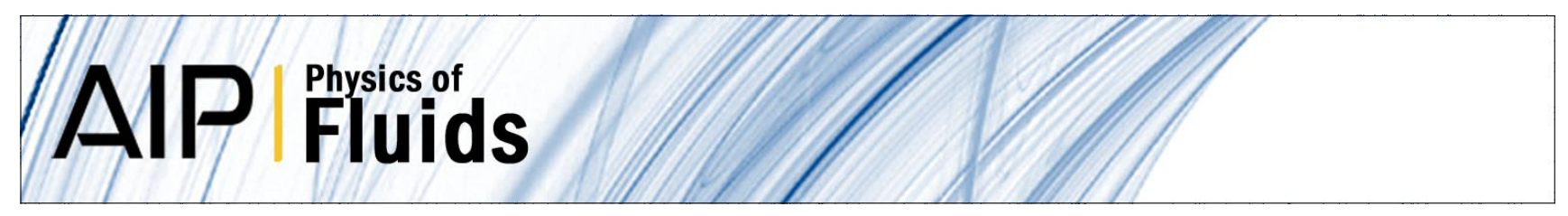

\title{
Angular distribution of energy spectrum in two-dimensional $\beta$-plane turbulence in the long-wave limit
}

\author{
Izumi Saito and Keiichi Ishioka
}

Citation: Phys. Fluids 25, 076602 (2013); doi: 10.1063/1.4813808

View online: http://dx.doi.org/10.1063/1.4813808

View Table of Contents: http://pof.aip.org/resource/1/PHFLE6/v25/i7

Published by the AIP Publishing LLC.

\section{Additional information on Phys. Fluids}

Journal Homepage: http://pof.aip.org/

Journal Information: http://pof.aip.org/about/about_the_journal

Top downloads: http://pof.aip.org/features/most_downloaded

Information for Authors: http://pof.aip.org/authors

\section{ADVERTISEMENT}

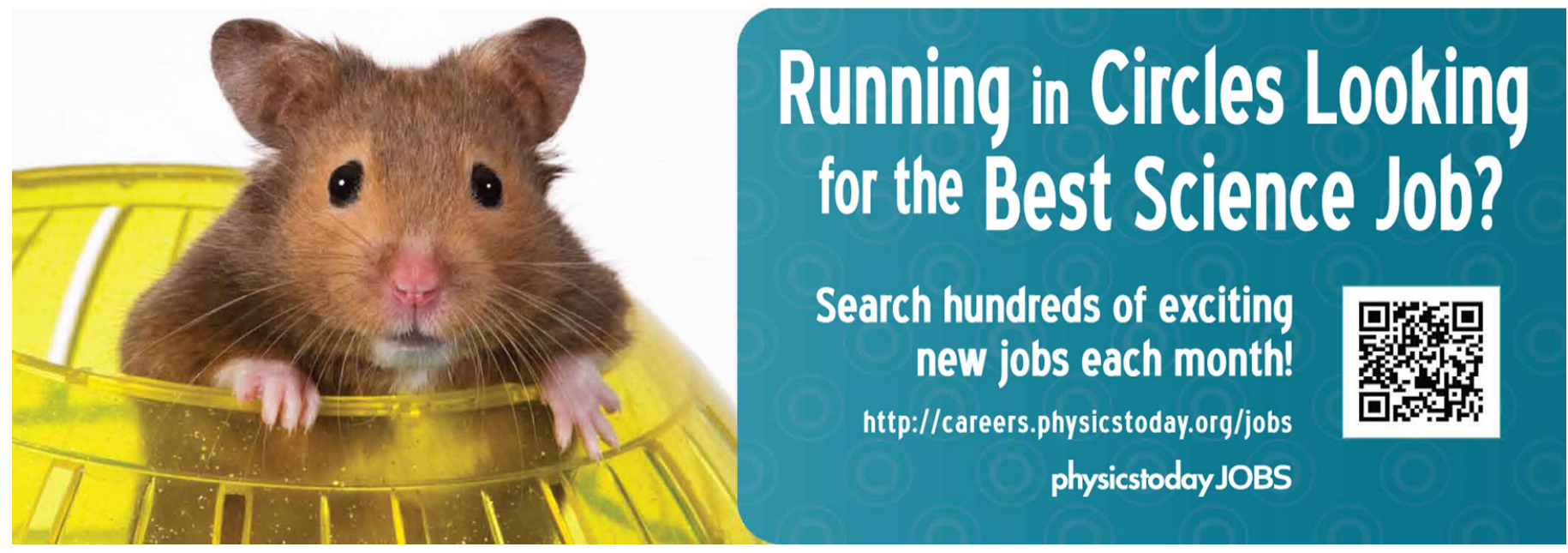


PHYSICS OF FLUIDS 25, 076602 (2013)

\title{
Angular distribution of energy spectrum in two-dimensional $\beta$-plane turbulence in the long-wave limit
}

\author{
Izumi Saito ${ }^{a)}$ and Keiichi Ishioka ${ }^{\text {b) }}$ \\ Division of Earth and Planetary Sciences, Graduate School of Science, Kyoto University, \\ Kyoto, Japan
}

(Received 6 December 2012; accepted 12 June 2013; published online 18 July 2013)

\begin{abstract}
The time-evolution of two-dimensional decaying turbulence governed by the longwave limit, in which $L_{\mathrm{D}} / L \rightarrow 0$, of the quasi-geostrophic equation is investigated numerically. Here, $L_{\mathrm{D}}$ is the Rossby radius of deformation, and $L$ is the characteristic length scale of the flow. In this system, the ratio of the linear term that originates from the $\beta$-term to the nonlinear terms is estimated by a dimensionless number, $\gamma=\beta L_{\mathrm{D}}^{2} / U$, where $\beta$ is the latitudinal gradient of the Coriolis parameter, and $U$ is the characteristic velocity scale. As the value of $\gamma$ increases, the inverse energy cascade becomes more anisotropic. When $\gamma \geq 1$, the anisotropy becomes significant and energy accumulates in a wedge-shaped region where $|l|>\sqrt{3}|k|$ in the twodimensional wavenumber space. Here, $k$ and $l$ are the longitudinal and latitudinal wavenumbers, respectively. When $\gamma$ is increased further, the energy concentration on the lines of $l= \pm \sqrt{3} k$ is clearly observed. These results are interpreted based on the conservation of zonostrophy, which is an extra invariant other than energy and enstrophy and was determined in a previous study. Considerations concerning the appropriate form of zonostrophy for the long-wave limit and a discussion of the possible relevance to Rossby waves in the ocean are also presented. (C) 2013 AIP Publishing LLC. [http://dx.doi.org/10.1063/1.4813808]
\end{abstract}

\section{INTRODUCTION}

As one of the simplest equations, the two-dimensional quasi-geostrophic vorticity equation on a $\beta$-plane,

$$
\frac{\partial}{\partial t}\left(\nabla^{2} \psi-\frac{1}{L_{\mathrm{D}}^{2}} \psi\right)+\beta \frac{\partial \psi}{\partial x}+\frac{\partial \psi}{\partial x} \frac{\partial \nabla^{2} \psi}{\partial y}-\frac{\partial \psi}{\partial y} \frac{\partial \nabla^{2} \psi}{\partial x}=0
$$

has been widely used to investigate large-scale flows in the atmosphere and in the ocean. Here, $t$ is the time, $(x, y)$ are the (eastward, northward) coordinates, $\psi(x, y, t)$ is the stream-function, $\beta$ is the $y$-derivative of the Coriolis parameter, and $L_{\mathrm{D}}$ is the Rossby radius of deformation. Turbulence governed by (1) with a dissipation term in the right-hand side exhibits a significant feature. As Rhines ${ }^{1}$ demonstrated, the energy cascade toward larger scales becomes anisotropic due to the second term (the $\beta$-term) in the left-hand side of (1), and the flow pattern tends to have a zonally elongated structure. This anisotropic energy cascade due to the $\beta$-term is now known as the Rhines effect.

In describing the Rhines effect, the characteristic length scale defined by $\beta$ and the velocity scale, $U$, as $L_{\beta}=\sqrt{U / \beta}$, plays an important role. This length scale is referred to as the

\footnotetext{
a) Author to whom correspondence should be addressed. Electronic mail: izumi@ kugi.kyoto-u.ac.jp. Telephone: +81-75-

753-3934. Fax: +81-75-753-3715. Present address: Kitashirakawa Oiwake-cho, Sakyo-ku, Kyoto 606-8502, Japan.

b)Electronic mail: ishioka@gfd-dennou.org
} 
Rhines scale. Letting $L$ be the length scale of the flow field, a nondimensional number $R h=$ $\left(L_{\beta} / L\right)^{2}=U /\left(\beta L^{2}\right)$ can be defined. The nondimensional number is referred to as the Rhines number and represents the ratio of the nonlinear terms in (1) to the linear $\beta$-term. When $R h$ $\gg 1$, i.e., $L \ll L_{\beta}$, the $\beta$-term is negligible and the dynamics is isotropic. If $R h \sim 1$, however, disturbances have the nature of Rossby waves, which leads to a dumbbell-shaped anisotropic energy spectrum in the two-dimensional wavenumber space, as demonstrated by Vallis and Maltrud. $^{2}$

The two papers cited above and many other studies have investigated (1) in the limit of $L_{\mathrm{D}} / L \rightarrow$ $\infty$. The case of a finite $L_{\mathrm{D}}$ was investigated extensively by Okuno and Masuda, ${ }^{3}$ who showed that the energy cascade becomes isotropic and the Rhines effect is suppressed in the case of $L<L_{\mathrm{D}} \ll$ $L_{\beta}$, whereas the Rhines effect still acts in the case of $L<L_{\beta}<L_{\mathrm{D}}$.

The other limit, $L_{\mathrm{D}} / L \rightarrow 0$, is also of interest. Based on Eq. (1) without the $\beta$-term, Larichev and McWilliams ${ }^{4}$ compared the time-evolutions of decaying turbulence in this limit (which they referred to as an asymptotic model) with those in the cases of finite but very small values of $L_{\mathrm{D}} / L$. They showed that the former exhibits essential features of the latter and that, in both cases, the onedimensional energy spectrum at large wavenumbers is approximately proportional to the negative sixth power of the wavenumber.

Retaining the $\beta$-term in the limit of $L_{\mathrm{D}} / L \rightarrow 0$ yields an equation, which is referred to as "the long-wave limit." For this long-wave limit, an invariant other than the basic invariants such as energy was discovered by Balk et al.,, and generalized later to the case of finite values of $L_{\mathrm{D}} / L$ by Balk. ${ }^{6}$ The extra invariant, which was referred to as zonostrophy by Nazarenko and Quinn, ${ }^{7}$ is approximately conserved when the nonlinearity of the system is weak. Based on the conservation of zonostrophy, Balk, ${ }^{8}$ Balk and Zakharov, ${ }^{9}$ and Balk et al. ${ }^{10}$ predicted that energy would accumulate in a wedge-shaped region $W$ in the two-dimensional wavenumber space,

$$
W=\{(k, l)|\sqrt{3}| k|<| l \mid\},
$$

in the course of time-evolution of turbulence if $L_{\mathrm{D}} / L \ll 1$. Here, $k$ and $l$ are the wavenumbers in the $x$ and $y$ directions, respectively. Very few numerical experiments, however, have been performed in order to verify this prediction. Connaughton et al. ${ }^{11}$ integrated (1) numerically with a forcing term and a dissipation term for the case of $L_{\mathrm{D}} / L \ll 1$. They demonstrated that energy diffuses along a contour line of the coefficient of zonostrophy (the coefficient will be shown in Sec. II) in the two-dimensional wavenumber space. This diffusion leads to energy accumulation around the lines of $\sqrt{3}|k|=|l|$. The results of a similar experiment were also reported by Nazarenko. ${ }^{12}$ Both of the above studies, however, examined time-evolution with a forcing that is localized in the twodimensional wavenumber space or from a localized initial wavenumber spectrum. Moreover, each of the two studies conducted numerical experiments only in a parameter range in which the nonlinearity of the system was very weak. Hence, it is still unclear that how weak the nonlinearity should be for the conservation of zonostrophy and for the appearance of anisotropy in the two-dimensional energy spectrum.

The primary purpose of the present study is to investigate the time-evolutions of decaying turbulence governed by the long-wave limit of (1) with a dissipation term from an isotropic initial energy spectrum in the two-dimensional wavenumber space with different degree of nonlinearity. In addition, we reconsider the asymptotic form of zonostrophy for this long-wave limit in order to interpret the experimental results and derive an appropriate form that has not appeared explicitly in the literature.

The remainder of the present paper is organized as follows. In Sec. II, we introduce the longwave limit of (1) and derive an appropriate asymptotic form of zonostrophy for this limit. In Sec. III, we conduct numerical experiments on decaying turbulence governed by the long-wave limit of (1) with a hyper-viscosity term and examine time-evolutions of the two-dimensional energy spectrum. Finally, Sec. IV presents a discussion and summary. 


\section{LONG-WAVE LIMIT OF THE QUASI-GEOSTROPHIC EQUATION AND ZONOSTROPHY FOR THIS LIMIT}

\section{A. Long-wave limit}

In this subsection, we first derive the long-wave limit of (1). Supposing that the length scale of the flow field, $L$, is much larger than the Rossby radius of deformation, $L_{\mathrm{D}}$, we formally expand the operator $\left(\nabla^{2}-L_{\mathrm{D}}^{-2}\right)$ as follows:

$$
\begin{aligned}
\left(\nabla^{2}-L_{\mathrm{D}}^{-2}\right) & =-L_{\mathrm{D}}^{-2}\left(1-L_{\mathrm{D}}^{2} \nabla^{2}\right) \\
& =-L_{\mathrm{D}}^{-2}\left(1+\left(L_{\mathrm{D}}^{2} \nabla^{2}\right)+\left(L_{\mathrm{D}}^{2} \nabla^{2}\right)^{2}+\cdots\right)^{-1} .
\end{aligned}
$$

Here, we assumed that $\nabla^{2}$ scales as $L^{-2}$ and that $L_{\mathrm{D}}^{2} \nabla^{2} \sim\left(L_{\mathrm{D}} / L\right)^{2} \ll 1$. Considering this expansion, multiplying $\left(1+\left(L_{\mathrm{D}}^{2} \nabla^{2}\right)+\left(L_{\mathrm{D}}^{2} \nabla^{2}\right)^{2}+\cdots\right)$ on both sides of $(1)$ yields

$$
\begin{gathered}
-\frac{1}{L_{\mathrm{D}}^{2}} \frac{\partial \psi}{\partial t} \\
+\beta\left(1+\left(L_{\mathrm{D}}^{2} \nabla^{2}\right)+\left(L_{\mathrm{D}}^{2} \nabla^{2}\right)^{2}+\cdots\right) \frac{\partial \psi}{\partial x} \\
+\left(1+\left(L_{\mathrm{D}}^{2} \nabla^{2}\right)+\left(L_{\mathrm{D}}^{2} \nabla^{2}\right)^{2}+\cdots\right)\left(\frac{\partial \psi}{\partial x} \frac{\partial \nabla^{2} \psi}{\partial y}-\frac{\partial \psi}{\partial y} \frac{\partial \nabla^{2} \psi}{\partial x}\right)=0 .
\end{gathered}
$$

If we omit $\left(L_{\mathrm{D}}^{2} \nabla^{2}\right)^{n}(n=1,2, \ldots)$ terms naively in (4), we obtain

$$
-\frac{1}{L_{\mathrm{D}}^{2}} \frac{\partial \psi}{\partial t}+\beta \frac{\partial \psi}{\partial x}+\frac{\partial \psi}{\partial x} \frac{\partial \nabla^{2} \psi}{\partial y}-\frac{\partial \psi}{\partial y} \frac{\partial \nabla^{2} \psi}{\partial x}=0
$$

The second term in the left-hand side of (5), however, only represents advection with constant velocity and has no effect on energy cascade if the domain is infinite or periodic. Thus, we should keep the $\left(L_{\mathrm{D}}^{2} \nabla^{2}\right)$ term in the second line in (4), which yields

$$
-\frac{1}{L_{\mathrm{D}}^{2}} \frac{\partial \psi}{\partial t}+\beta \frac{\partial \psi}{\partial x}+\beta L_{\mathrm{D}}^{2} \frac{\partial \nabla^{2} \psi}{\partial x}+\frac{\partial \psi}{\partial x} \frac{\partial \nabla^{2} \psi}{\partial y}-\frac{\partial \psi}{\partial y} \frac{\partial \nabla^{2} \psi}{\partial x}=0 .
$$

Considering that the second term in the left-hand side of (6) only represents constant advection, as stated above, we remove this term by a suitable Galilean transform, which was also used in Okuno and Masuda, ${ }^{3}$ and finally obtain

$$
-\frac{1}{L_{\mathrm{D}}^{2}} \frac{\partial \psi}{\partial t}+\beta L_{\mathrm{D}}^{2} \frac{\partial \nabla^{2} \psi}{\partial x}+\frac{\partial \psi}{\partial x} \frac{\partial \nabla^{2} \psi}{\partial y}-\frac{\partial \psi}{\partial y} \frac{\partial \nabla^{2} \psi}{\partial x}=0 .
$$

Here, the $x$ coordinate has been replaced with the moving frame. We hereinafter refer to (7) as the long-wave limit.

\section{B. Nondimensionalization}

We nondimensionalize (7) in the following manner:

$$
\begin{array}{r}
x=L x_{*}, \quad y=L y_{*}, \quad \psi=U L \psi_{*}, \\
t=\frac{L^{3}}{L_{\mathrm{D}}^{2} U} t_{*}, \quad \nabla^{2}=L^{-2} \nabla_{*}^{2} .
\end{array}
$$


Here, $L$ and $U$ are the characteristic length and velocity scales, respectively, and variables with $*$ indicate nondimensional variables. Then, we have

$$
-\frac{\partial \psi_{*}}{\partial t_{*}}+\gamma \frac{\partial}{\partial x_{*}} \nabla_{*}^{2} \psi_{*}+\frac{\partial \psi_{*}}{\partial x_{*}} \frac{\partial \nabla_{*}^{2} \psi_{*}}{\partial y_{*}}-\frac{\partial \psi_{*}}{\partial y_{*}} \frac{\partial \nabla_{*}^{2} \psi_{*}}{\partial x_{*}}=0,
$$

where

$$
\gamma=\frac{\beta L_{\mathrm{D}}^{2}}{U}
$$

is a dimensionless number. This dimensionless number, which was first introduced by Okuno and Masuda, ${ }^{3}$ who referred to the reciprocal of $\gamma$ as $\alpha$, gives a rough estimate of the ratio of the linear term originating from the $\beta$-term to the nonlinear terms. We hereinafter refer to the second term in the left-hand side of (9) as the $\gamma$-term. Note that, in contrast to the Rhines number, the number $\gamma$ does not depend on the length scale $L$.

\section{Representation in the wavenumber space}

Let us assume that the domain is infinite and the nondimensionalized streamfunction can be represented by the inverse Fourier transform as follows:

$$
\psi_{*}\left(x_{*}, y_{*}, t_{*}\right)=\frac{1}{2 \pi} \int_{-\infty}^{\infty} \int_{-\infty}^{\infty} \hat{\psi}_{\mathbf{k}}\left(t_{*}\right) \mathrm{e}^{\mathrm{i}\left(k x_{*}+l y_{*}\right)} \mathrm{d} k \mathrm{~d} l .
$$

Here, $\mathbf{k}=(k, l)$ is the wavenumber vector. Then, the Fourier transform of the nondimensionalized long-wave limit equation (9) yields

$$
\mathrm{i} \frac{\partial \hat{\psi}_{\mathbf{k}}}{\partial t_{*}}=\omega_{\mathbf{k}} \hat{\psi}_{\mathbf{k}}+\mathrm{i} \iiint \int W_{\mathbf{k}_{1}, \mathbf{k}_{2}} \delta\left(-\mathbf{k}+\mathbf{k}_{1}+\mathbf{k}_{2}\right) \hat{\psi}_{\mathbf{k}_{1}} \hat{\psi}_{\mathbf{k}_{2}} \mathrm{~d} \mathbf{k}_{1} \mathrm{~d} \mathbf{k}_{2} .
$$

Here, $\delta$ is the Dirac delta function,

$$
\omega_{\mathbf{k}}=\gamma|\mathbf{k}|^{2} k
$$

is the frequency of the Rossby wave of wavenumber $\mathbf{k}$, and

$$
W_{\mathbf{k}_{1}, \mathbf{k}_{2}}=\frac{1}{4 \pi}\left(k_{1} l_{2}-k_{2} l_{1}\right)\left(\left|\mathbf{k}_{2}\right|^{2}-\left|\mathbf{k}_{1}\right|^{2}\right)
$$

is the interaction coefficient that arises from the nonlinear terms.

In the system governed by (12), there are two fundamental invariants, which are defined as

$$
E=\iint \varepsilon_{\mathbf{k}} \mathrm{d} \mathbf{k} \quad \text { and } \quad \Omega=\iint|\mathbf{k}|^{2} \varepsilon_{\mathbf{k}} \mathrm{d} \mathbf{k},
$$

where

$$
\varepsilon_{\mathbf{k}}=\frac{1}{2}\left|\hat{\psi}_{\mathbf{k}}\right|^{2} .
$$

The former $(E)$ is the potential energy and the latter $(\Omega)$ is the kinetic energy. In the present paper, however, we hereinafter refer to $E$ and $\Omega$ simply as energy and enstrophy, respectively, because $E$ corresponds to the total energy, and $\Omega$ to the potential enstrophy when $L_{\mathrm{D}} / L$ is finite.

\section{Asymptotic form of zonostrophy}

The two invariants defined above have the form of

$$
M=\iint \phi_{\mathbf{k}} \varepsilon_{\mathbf{k}} \mathrm{d} \mathbf{k} .
$$

Here, $\phi_{\mathbf{k}}$ is the coefficient that defines the quantity $M$. Energy $(E)$ and enstropy $(\Omega)$ correspond to $M$, with $\phi_{\mathbf{k}}$ chosen as

$$
\phi_{\mathbf{k}}=1 \quad \text { and } \quad \phi_{\mathbf{k}}=|\mathbf{k}|^{2} \text {, }
$$


respectively. Zonostrophy is another quantity that has the form of (17) and is approximately conserved when the nonlinearity of (1) (or (7) in the long-wave limit) is sufficiently weak. In order to (at least approximately) conserve $M, \phi_{\mathbf{k}}$ must be written as follows:

$$
\phi_{\mathbf{k}}=\frac{\eta_{\mathbf{k}}}{k}
$$

where $\eta_{\mathbf{k}}$ is a function of $\mathbf{k}$ that satisfies

$$
\eta_{\mathbf{k}}+\eta_{\mathbf{k}_{1}}+\eta_{\mathbf{k}_{2}}=0
$$

whenever

$$
\mathbf{k}+\mathbf{k}_{1}+\mathbf{k}_{2}=\mathbf{0} \text { and } \omega_{\mathbf{k}}+\omega_{\mathbf{k}_{1}}+\omega_{\mathbf{k}_{2}}=0 .
$$

It is easy to check that $\phi_{\mathbf{k}}$ corresponding to $E$ or $\Omega$ satisfies this condition. When a finite $L_{\mathrm{D}} / L$ is considered and the length scale is nondimensionalized by $L_{\mathrm{D}}$, the definition of $\omega_{\mathbf{k}}$ should be replaced by $\omega_{\mathbf{k}}=-k /\left(1+|\mathbf{k}|^{2}\right)$ rather than (13) and (18) should be replaced by $\phi_{\mathbf{k}}=\eta_{\mathbf{k}} / \omega_{\mathbf{k}}$. In this case, the explicit form of $\eta_{\mathbf{k}}$ for zonostrophy is given as

$$
\eta_{\mathbf{k}}=\arctan \left(\frac{l-k \sqrt{3}}{|\mathbf{k}|^{2}}\right)-\arctan \left(\frac{l+k \sqrt{3}}{|\mathbf{k}|^{2}}\right) .
$$

For the derivation of (21) and the reason why zonostrophy is approximately conserved, refer to Balk $^{6}$ and Balk and van Heerden. ${ }^{13}$

For zonostrophy in the long-wave limit, an explicit form of $\eta_{\mathbf{k}}$ was given by Balk et al..$^{5}$ as

$$
\eta_{\mathbf{k}}=\frac{k^{3}}{l^{2}-3 k^{2}}
$$

This form of $\eta_{\mathbf{k}}$, however, gives $\phi_{\mathbf{k}}$, which changes sign, and the use of the zonostrophy defined by this $\eta_{\mathbf{k}}$ in interpreting the experimental results presented later herein is not straightforward. In order to see how crucial the sign-definiteness is, let us suppose that $\phi_{\mathbf{k}}$ of enstrophy had a negative value at a certain $\mathbf{k}=\mathbf{k}^{\prime}$ for example. Then, the conservation of enstrophy would fail to explain the inverse energy cascade because in this improbable case, arbitrary amount of energy can move to larger wavenumber regions by depositing proper amount of energy at $\mathbf{k}^{\prime}$ so that enstrophy is conserved. Similar difficulty is encountered if we use the zonostrophy defined by the sign-indefinite coefficient as (22).

Reexamining the long-wave limit of (21), we found that the following form of $\eta_{\mathbf{k}}$,

$$
\eta_{\mathbf{k}}=\left\{\begin{array}{rl}
1 & (|l|<\sqrt{3} k) \\
-1 & (|l|<-\sqrt{3} k) \\
0 & (|l|>\sqrt{3}|k| \text { or }|\mathbf{k}|=0) \\
1 / 2(|l| & =\sqrt{3} k \text { and }|\mathbf{k}| \neq 0) \\
-1 / 2(|l| & =-\sqrt{3} k \text { and }|\mathbf{k}| \neq 0)
\end{array},\right.
$$

is a more straightforward form for the limit. The derivation of this form as the long-wave limit of (21) and proof that this $\eta_{\mathbf{k}}$ satisfies (19) whenever (20) holds is presented in Appendix. This form of $\eta_{\mathbf{k}}$ gives $\phi_{\mathbf{k}}$ through (18) as follows:

$$
\phi_{\mathbf{k}}=\left\{\begin{array}{rl}
1 /|k| & (|l|<\sqrt{3}|k|) \\
0 & (|l|>\sqrt{3}|k| \text { or }|\mathbf{k}|=0) \\
1 /(2|k|) & (|l|=\sqrt{3}|k| \text { and }|\mathbf{k}| \neq 0)
\end{array} .\right.
$$

Figure 1 shows the distribution of this $\phi_{\mathbf{k}}$ in the wavenumber space. The coefficient, $\phi_{\mathbf{k}}$, is equal to zero in the region $W$, which is defined as (2), but increases in proportional to $|k|^{-1}$ toward small 


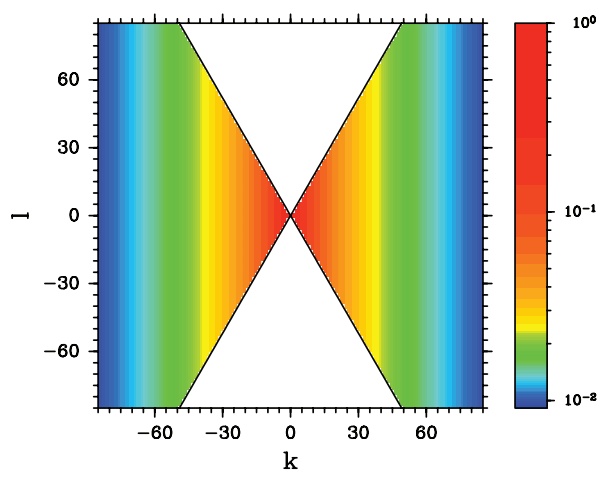

FIG. 1. Distribution of $\phi_{\mathbf{k}}$ defined by (24) in the two-dimensional wavenumber space. The region in which $\phi_{\mathbf{k}}=0$ is not colored. The two lines that bound the zero-valued region are $l= \pm \sqrt{3} k$.

wavenumbers outside of the region $W$. This $\phi_{\mathbf{k}}$ is non-negative, and the zonostrophy defined by $\phi_{\mathbf{k}}$,

$$
Z=\iint \phi_{\mathbf{k}} \varepsilon_{\mathbf{k}} \mathrm{d} \mathbf{k}
$$

is useful for interpreting the experimental results presented later herein. We hereinafter refer to this $Z$ simply as the zonostrophy.

\section{NUMERICAL EXPERIMENTS}

\section{A. Experimental setup}

In this section, we conduct numerical experiments to investigate the time-evolutions of decaying turbulence in the long-wave limit. As the basic equation, we use the following equation:

$$
-\frac{\partial \psi}{\partial t}+\gamma \frac{\partial}{\partial x} \nabla^{2} \psi+\frac{\partial \psi}{\partial x} \frac{\partial \nabla^{2} \psi}{\partial y}-\frac{\partial \psi}{\partial y} \frac{\partial \nabla^{2} \psi}{\partial x}=(-1)^{p} v_{p} \nabla^{2 p} \psi
$$

rather than (9). Here, we have omitted $*$ for dimensionless variables, and the right-hand side of (26) is a hyper-viscosity term introduced in order to avoid spurious accumulation of enstrophy near the truncation wavenumber for the computation.

We impose a $2 \pi$-periodic boundary condition in both the $x$ and $y$ directions and adopt the Fourier spectral method with a truncation wavenumber of $K_{T}=85$ for the spatial discretization. The nonlinear terms are computed using the transform method with alias-free grids of $256 \times 256$. The time integration scheme is the classical fourth-order Runge-Kutta scheme with time step $\Delta t=2 \times 10^{-7}$. We set the order of hyper-viscosity $p=10$ and the hyper-viscosity coefficient $v_{p}=5 \times 10^{-35}$ in the following computations. In time-integrations, we use the following transform of the dependent variable

$$
\hat{\psi}_{\mathbf{k}}(t)=\hat{\Psi}_{\mathbf{k}}(t) \exp \left(-\mathrm{i} \gamma|\mathbf{k}|^{2} k t-v_{p}|\mathbf{k}|^{2 p} t\right)
$$

in the wavenumber space in order to extend the time-step size.

The initial condition is a random stream function field, the one-dimensional energy spectrum $E_{K}$ of which is given as follows:

$$
E_{K} \propto\left(\frac{2}{\sqrt{K / K_{0}}+\sqrt{K_{0} / K}}\right)^{m},
$$


where $K=|\mathbf{k}|=\sqrt{k^{2}+l^{2}}$ is the total wavenumber. We set $K_{0}=20$ and $m=1000$. The phase of each Fourier component is set randomly. The total energy of the initial state is set to $1 / 800$, which means that the root-mean-square velocity $\left(u_{0}\right)$ for the initial state is approximately

$$
u_{0}=\sqrt{2 \Omega_{(t=0)}} \approx K_{0} \sqrt{2 E_{(t=0)}}=1 .
$$

In other words, we have chosen the initial root-mean-square velocity as the characteristic velocity $(U)$ for the nondimensionalization. On the other hand, since we have set $K_{0}=20$, the length scale for the nondimensionalization differs from the length scale of the initial flow field. The nondimensional parameter $\gamma$ is, however, independent of the length scale, as stated in Subsection II B. Thus, if the results for the case in which the length scale of the initial flow is chosen as the length scale for the nondimensionalization are desired, the time scale of the following results need only be translated appropriately.

In Subsection III B, we present the results for $\gamma=0,0.25,1,5,20$. For each experiment, the numerical time integration of (26) is conducted until $t=0.4$. This integration period is 80 times the initial characteristic advection time, which is estimated to be $1 /\left(u_{0} K_{0}\right)=0.05$.

\section{B. Results}

In order to compare the obtained results with the results of Larichev and McWilliams, ${ }^{4}$ let us first examine the one-dimensional energy spectra. Figure 2 shows the one-dimensional energy spectra for the initial state and for the final states of time-evolutions for five values of $\gamma$. For each experiment, the energy spectrum broadens with time and gradually shifts its peak toward smaller wavenumbers. (The details for the time-evolutions of the energy spectra are not shown.) In the case of $\gamma=0$, the governing equation is almost the same as that used in Larichev and McWilliams ${ }^{4}$ except for the hyper-viscosity term, and the shape of the spectrum finally becomes approximately $E_{K} \propto K^{-5}$ in the large- $K$ region of $7 \leq K \leq 40$ (solid red curve). This energy spectrum is slightly more shallow than that obtained by Larichev and McWilliams ${ }^{4}\left(E_{K} \propto K^{-6}\right)$ for their asymptotic model. This difference may have arisen from the difference in the hyper-viscosity term. For larger values of $\gamma$, the $\gamma$-term in the left-hand side of (26) comes into play. As shown below, the $\gamma$-term not only makes the two-dimensional energy spectrum anisotropic but also slows down the inverse energy cascade. The larger the values of $\gamma$, the more energy remains in the large- $K$ region. In particular, in the case of $\gamma=20$, a large amount of energy remains around the initial energy peak at $K=K_{0}=20$.

The difference in the time-evolutions of energy spectra depending on the parameter $\gamma$ can be seen more clearly in two-dimensional energy spectra. Figure 3 shows two-dimensional energy spectra for the initial state and those for the final states for the five values of $\gamma$. For each value of $\gamma$, we conducted 41 experiments, changing the initial random choice of the stream-function field, and created the figure of the two-dimensional energy spectrum by taking the ensemble average. The

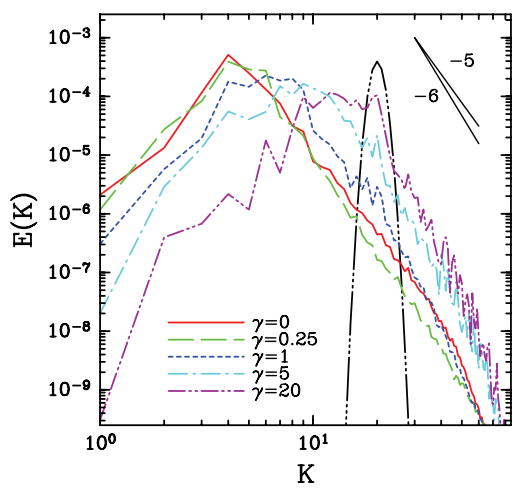

FIG. 2. One-dimensional energy spectra at $t=0$ (dashed-three-dotted black curve) and at $t=0.4$ for $\gamma=0$ (solid red), $\gamma=$ 0.25 (long dashed green), $\gamma=1$ (short dashed blue), $\gamma=5$ (dashed-dotted cyan), and $\gamma=20$ (dashed-two-dotted purple). 
(a)

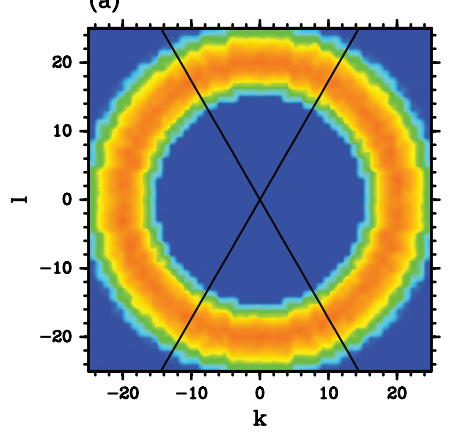

(d)

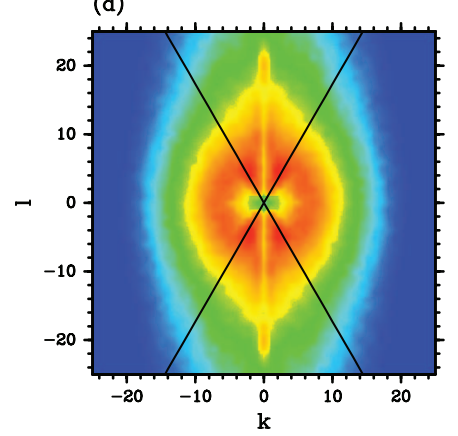

(b)

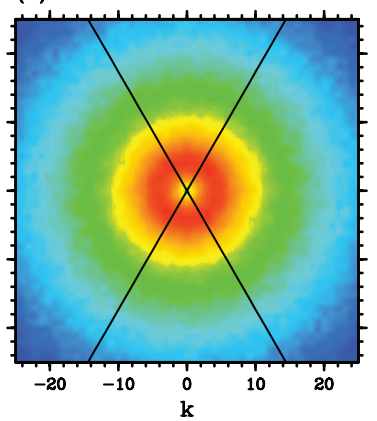

(e)

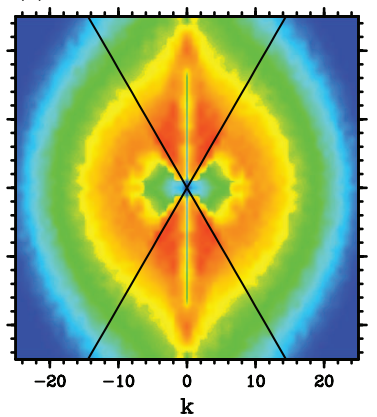

(c)

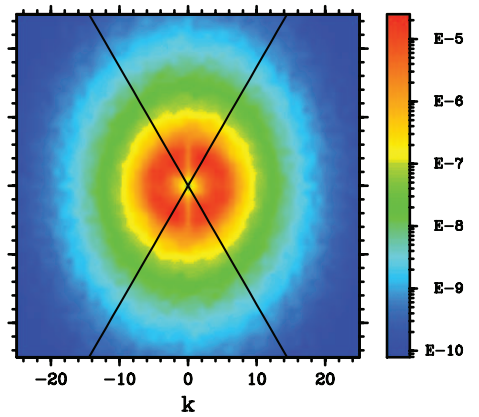

(f)

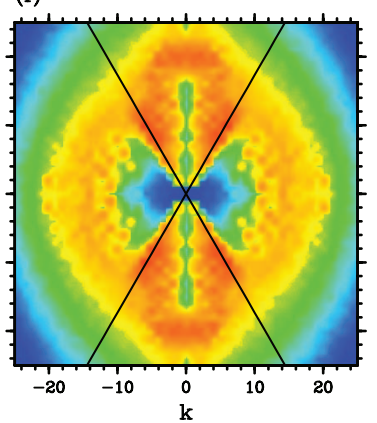

FIG. 3. (a) Two-dimensional energy spectrum at $t=0$ within a rectangular domain of $|k|,|l| \leq 25$ averaged over 41 ensemble members. (b)-(f) Same as (a) except that at $t=0.4$ for $\gamma=0,0.25,1,5$, and 20, respectively. E- $x$ indicates $10^{-x}$. In each figure, the lines of $l= \pm \sqrt{3} k$ are drawn for reference.

initial spectrum is isotropic (Fig. 3(a)) as given, and the spectrum for the final state for the $\gamma=0$ case remains isotropic after the inverse energy cascade (Fig. 3(b)). For the cases of non-zero values of $\gamma$, however, the $\gamma$-term makes the energy cascade anisotropic. Even in the case of $\gamma=0.25$, the anisotropy in the two-dimensional energy spectrum can be seen (Fig. 3(c)). The ring-shaped peak near the origin is severed by the region of relatively low energy density around the $l$-axis, and the energy distribution in larger wavenumbers is slightly elongated along the $l$-axis. In the case of $\gamma=1$, which means that the $\gamma$-term in the left-hand side of (26) has an amplitude of the same order as the nonlinear terms have in the initial state, the anisotropy is more prominent (Fig. 3(d)). As another feature, two peaks near $(k, l)=(0, \pm 20)$ can be also seen. These peaks are merely remnants of the initial energy distribution. For larger values of $\gamma$, in addition to the anisotropy described above, the slit-like region near the $l$-axis and the elongated structure of the energy spectrum, the significance of the wedge-shaped region $W$ defined by (2) also becomes apparent (Fig. 3(e) for $\gamma=5$ and Fig. 3(f) for $\gamma=20$ ). In both the figures, the energy accumulation in the wedge-shaped region is clear in the smaller-wavenumber region, although the slit-like region of low energy density near the $l$-axis is still prominent. In contrast, a hollow region in which the energy density is very low appears outside the wedge-shaped region. The contrast across the boundary (the lines of $l= \pm \sqrt{3} \mathrm{k}$ ) of the wedge-shaped region is more significant in the case of $\gamma=20$ (Fig. 3(f)). The energy accumulation in the wedge-shaped region (and the corresponding energy escape from outside of the wedge-shaped region) is consistent with the theoretical prediction given by the previous studies. ${ }^{8-10}$ Assuming the conservation of zonostrophy defined by (25), the accumulation can be interpreted as follows. As defined by (24), the coefficient $\phi_{\mathbf{k}}$ increases as $|k|$ decreases outside of the wedge-shaped region. In order to conserve zonostrophy during the inverse energy cascade, some amount of energy must escape from outside of the wedge-shaped region into the wedge-shaped region, in which the coefficient $\phi_{\mathbf{k}}=0$ and zonostrophy conservation does not impose a barrier on the inverse energy cascade. 


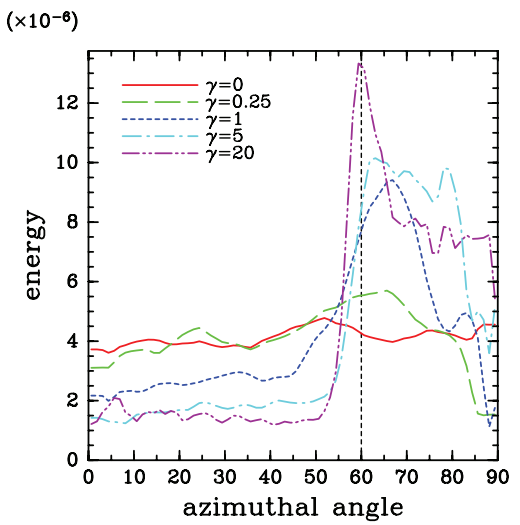

FIG. 4. Angular distributions of energy spectra corresponding to the final $(t=0.4)$ states shown as Figs. 3(b)-3(f). The solid red curve corresponds to Fig. 3(b) $(\gamma=0)$, the long dashed green curve corresponds to Fig. 3(c) $(\gamma=0.25)$, the short dashed blue curve corresponds to Fig. 3(d) $(\gamma=1)$, the dashed-dotted cyan curve corresponds to Fig. 3(e) $(\gamma=5)$, and the dashed-two-dotted purple curve corresponds to Fig. 3(f) $(\gamma=20)$.

The importance of the wedge-shaped region can be emphasized by examining the angular distribution of the energy density. Figure 4 shows the angular distribution of the energy density for the final states for five values of $\gamma$. Here, as in Fig. 3, the ensemble average has been taken, and the azimuthal angle $\theta$ is defined as $\theta=\tan ^{-1}(|l / k|)$. While the spectrum is isotropic in the case of $\gamma=0$ (solid red curve), the case of $\gamma=0.25$ (long dashed green curve) yields a low-density region around $\theta=90^{\circ}$. As the value of $\gamma$ increases $(\gamma=1,5$, and 20; short dashed blue, dashed-dotted cyan, and dashed-two-dotted purple curves, respectively), more energy accumulates in the region of $\theta>60^{\circ}$, which corresponds to the wedge-shaped region in the two-dimensional wavenumber space. Furthermore, in the case of the largest value of $\gamma(\gamma=20$; dashed-two-dotted purple curve), the energy concentration around $\theta=60^{\circ}$ is significant. A hint of this concentration can even be seen for the cases in which $\gamma=1$ (short dashed blue curve) and $\gamma=5$ (dashed-dotted cyan curve).

Since the energy cascade is highly anisotropic in the case of $\gamma=20$, as shown above, let us examine the time-evolution of the two-dimensional energy spectrum (Fig. 5). From the initial isotropic distribution (Fig. 5(a)), an inverse energy cascade is first observed primarily in the wedgeshaped region (Fig. 5(b)). After that, however, the energy density increases around the lines of $l= \pm \sqrt{3} k$, keeping energy from leaving the wedge-shaped region (Figs. 5(c)-5(e)). Finally, the peak energy density shifts toward a smaller wavenumber outside the wedge-shaped region, allowing some amount of energy to escape into the wedge-shaped region (Fig. 5(f)).

The energy concentration around the lines of $l= \pm \sqrt{3} k$ in the two-dimensional energy spectrum, which corresponds to the peak near $\theta=60^{\circ}$ in the angular energy distribution shown in Fig. 4, is discernible even in the physical space. Figure 6 shows the stream-function fields for the initial state (Fig. 6(a)) and for the final states for five values of $\gamma$ (Figs. 6(b)-6(f)). The initial fine structures (Fig. 6(a)) evolve into a larger-scale structure (Fig. 6(b)), corresponding to the inverse energy cascade. Figure 6(b), however, does not exhibit anisotropy. Even in the case of a non-zero but small value of $\gamma(\gamma=0.25$; Fig. 6(c)), no significant anisotropy can be seen. As the value of $\gamma$ increases, the final state tends to have a smaller structure and a more significant anisotropy $(\gamma=1$, 5, and 20; Figs. 6(d)-6(f), respectively). In particular, in the case of the largest $\gamma(\gamma=20$; Fig. 6(f)), a structure parallel to the lines of $x= \pm \sqrt{3} y$ is clearly observed, which corresponds to the energy concentration around the lines of $l= \pm \sqrt{3} k$ in the two-dimensional energy spectrum.

As described above, we assumed the conservation of zonostrophy in order to interpret the importance of the wedge-shaped region. Let us examine how well zonostrophy, energy, and enstrophy are conserved in the numerical time-evolutions. Figure 7 shows the time-evolutions of energy (Fig. 7(a)), enstrophy (Fig. 7(b)), and zonostrophy (Fig. 7(c)) for five values of $\gamma$. Due to the hyperviscosity term introduced in the right-hand side of (26), both energy and enstrophy decrease with 
(a)

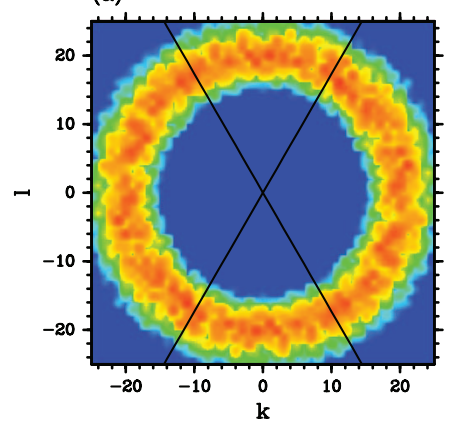

(d)

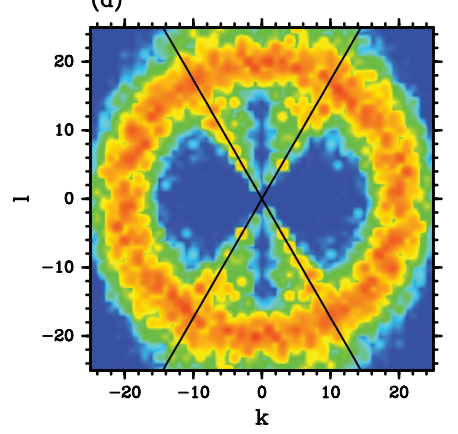

(b)

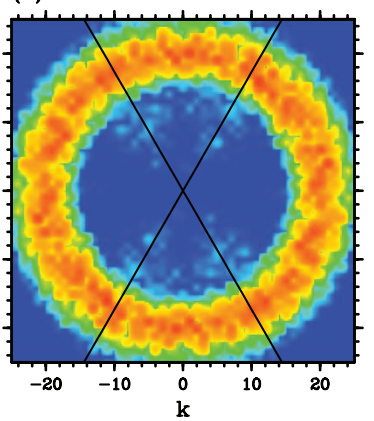

(e)

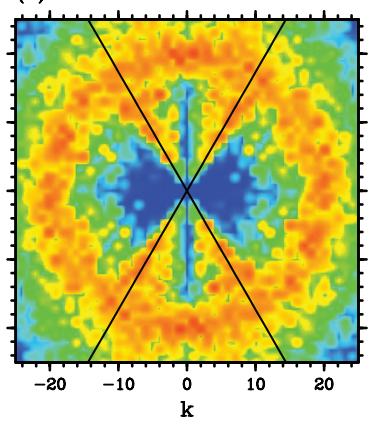

(c)

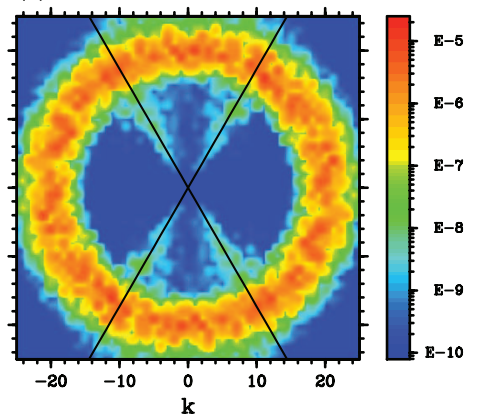

(f)

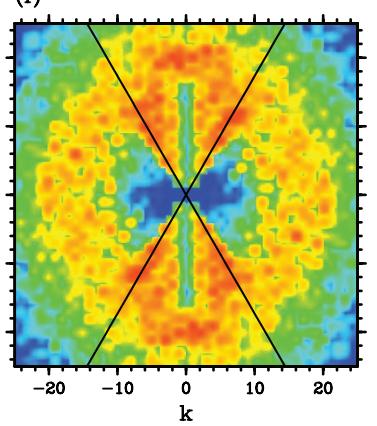

FIG. 5. Snapshots of two-dimensional energy spectra within a rectangular domain of $|k|,|l| \leq 25$ in the time-evolution for the case in which $\gamma=20$. (a) At $t=0$, (b) at $t=1.2 \times 10^{-4}$, (c) at $t=4 \times 10^{-4}$, (d) at $t=8 \times 10^{-3}$, (e) at $t=0.04$, and (f) at $t=0.2$. $\mathrm{E}-x$ indicates $10^{-x}$. In each figure, the lines of $l= \pm \sqrt{3} k$ are drawn for reference. Note that, unlike Fig. 3, these figures show the time-evolution of one member of the ensemble experiments.

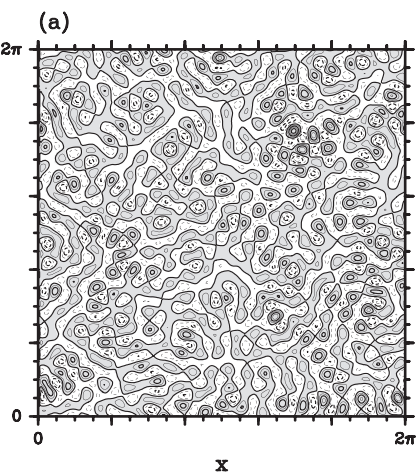

(d)

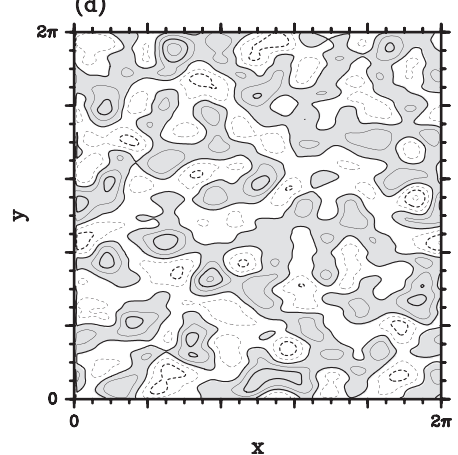

(b)

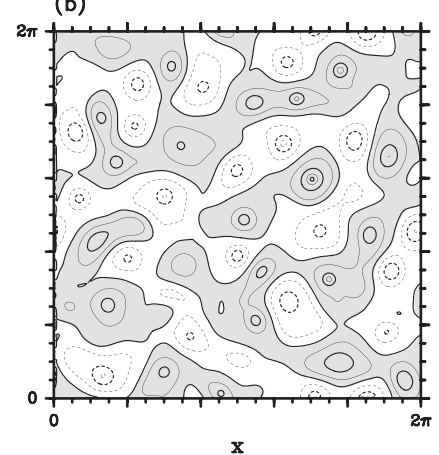

(e)

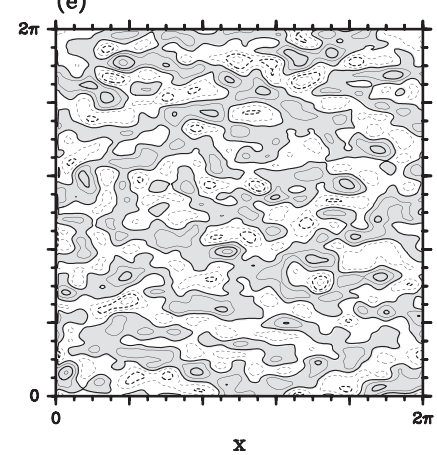

(c)

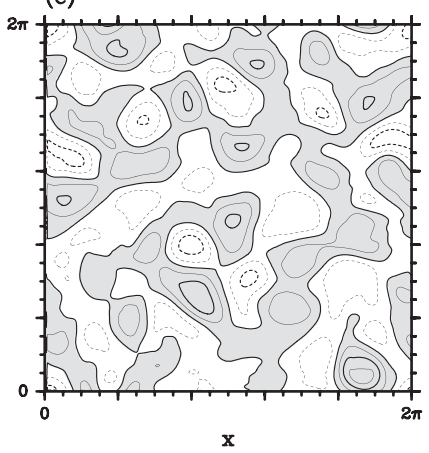

(f)

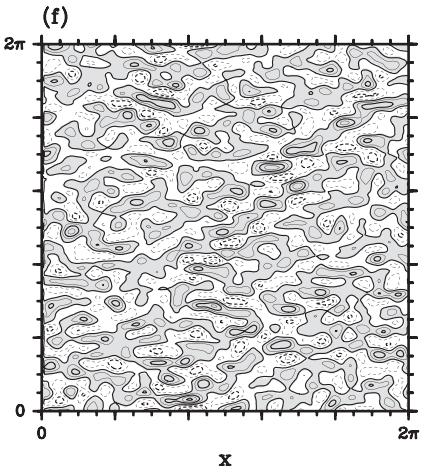

FIG. 6. Stream function fields. (a) for the initial state. (b)-(f) At $t=0.4$ for $\gamma=0,0.25,1,5$, and 20, respectively. The contour interval is 0.05 , and positive regions are shaded. 

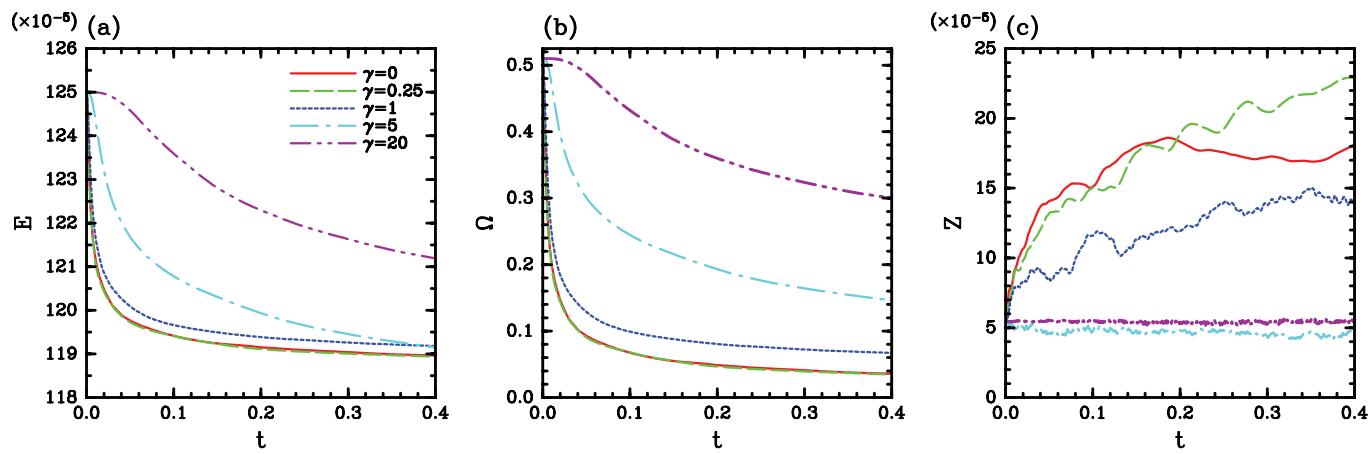

FIG. 7. Time-evolutions of (a) energy $(E)$, (b) enstrophy $(\Omega)$, and (c) zonostrophy $(Z)$. The results for $\gamma=0,0.25,1,5,20$ are drawn by solid red, long dashed green, short dashed blue, dashed-dotted cyan, and dashed-two-dotted purple curves, respectively.

time for all values of $\gamma$ (Figs. 7(a) and 7(b)). This decrease, however, depends on the value of $\gamma$. As shown in Figs. 7(a) and 7(b), this decrease becomes smaller as $\gamma$ increases. This is interpreted as follows. In the case of larger values of $\gamma(\gamma=5$ (dashed-dotted cyan curve) and $\gamma=20$ (dashedtwo-dotted purple curve)), the inverse energy cascade is slowed by the conservation of zonostrophy, which in turn slows the enstrophy cascade to larger wavenumbers, which reduces the dissipation of enstrophy by the hyper-viscosity term. Zonostrophy conservation for larger $\gamma$ cases can be confirmed by referring to Fig. 7(c). While the value of zonostrophy changes significantly for small $\gamma$ cases $(\gamma=0,0.25$, and 1 ; solid red, long dashed green, and short dashed blue curves, respectively), zonostrophy is well conserved for the larger values of $\gamma(\gamma=5$ (dashed-dotted cyan curve) and $\gamma=20$ (dashed-two-dotted purple curve)). This appears to be a natural result because the conservation of zonostrophy holds when the nonlinearity of the basic equation is sufficiently weak.

Before closing this section, let us mention two issues concerning the validity of numerical experiments conducted in the present study. First issue is about equilibration. Since energy and enstrophy are still decreasing even in the late stage of the time-evolution (near $t=0.4$ in Figs. 7(a) and 7(b)), some readers may wonder whether or not lack of equilibration affects the anisotropy described above especially for large values of $\gamma(\gamma=5$ and 20). For each value of $\gamma$, we conducted additional experiments in which the governing equation was integrated further until $t$ $=2.4$, which is 6 times longer than the integration period of the experiments described above, and confirmed that the nature of the anisotropy of the two-dimensional energy spectrum did not change qualitatively with time from $t=0.4$ to $t=2.4$ (not shown) though the peak of one-dimensional energy spectrum moved slightly to smaller wavenumber regions. It is expected that the nature of the anisotropy will not change qualitatively even after $t=2.4$ because, unlike the well-known Rhines number, the parameter $\gamma$ does not depend on the length scale. Second issue is about the spatial resolution. Although the choice of resolution $K_{\mathrm{T}}=85(256 \times 256$ grids $)$ may seem rather low for the integration of the simple equation (26), it should be noted that maximum frequency of Rossby waves in this system is $2 \gamma K_{\mathrm{T}}^{3}$ and such a high frequency requires a very small time step, which makes it difficult to conduct many numerical experiments of a high resolution and a long time-integration period. In the present paper, we chose a relatively low resolution of $K_{\mathrm{T}}=85$ in order to conduct ensemble numerical experiments, changing the initial random field of the stream function and the parameter $\gamma$, which made it possible to obtain a clear image of the anisotropy of the energy cascade in the average view of the two-dimensional energy spectrum (Figs. 3 and 4). Some readers also wonder whether or not the choice of resolution can affect how well zonostrophy is conserved. The spatial resolution used in the present paper is, however, not too low to investigate the conservation of zonostrophy. This is because the coefficient for zonostrophy, $\phi_{\mathbf{k}}$, is large in small wavenumber region compared to that for energy since $\phi_{\mathbf{k}} \sim O\left(|k|^{-1}\right)$ as defined by (17). As seen in Fig. 7(a), energy is conserved well and keeps more than $95 \%$ of its initial value even with the hyper-viscosity. Hence, the truncation and the hyper-viscosity is thought to have little influence on whether or not zonostrophy is well conserved. In fact, we conducted several experiments, increasing the spatial 
resolution to $K_{\mathrm{T}}=170(512 \times 512$ grids $)$ and confirmed that there were no qualitative differences in how well zonostrophy was conserved and in the nature of the anisotropy in the two-dimensional energy spectrum (not shown). As for the slope of the one-dimensional energy spectrum, however, the experiments of the higher spatial resolution shows a slight dependence of the slope on the spatial resolution. This indicates that experiments with a higher resolution and a more careful choice of hyper-viscosity are required to determine the slope of the one-dimensional energy spectrum in this system. However, we do not pursue this issue further since the primary subjects of the present study are the conservation of zonostrophy and the appearance of the anisotropy of the two-dimensional energy spectrum.

\section{SUMMARY AND DISCUSSION}

In the present paper, we numerically investigated the time-evolution of decaying turbulence that is governed by the long-wave limit of the two-dimensional quasi-geostrophic equation. As shown in Sec. III, the features of the time-evolution differ significantly depending on the parameter $\gamma$, which determines the relative importance of the $\gamma$-term in the left-hand side of (26) compared with the nonlinear terms. If $\gamma$ is non-zero, the inverse energy cascade exhibits anisotropy. When $\gamma \geq 1$, the anisotropy becomes significant and energy accumulation in the wedge-shaped region, which is defined by (2), is clearly observed in the two-dimensional wavenumber space. This energy accumulation has been predicted theoretically in the previous studies, ${ }^{8-10}$ based on the conservation of zonostrophy. To our knowledge, the present paper describes the first study to show that energy accumulation occurs in the time-evolution of decaying turbulence from an isotropic initial condition in the long-wave limit, although the accumulation has been reported for small but finite values of $L_{\mathrm{D}} / L$ with localized initial energy spectra. ${ }^{11,12}$ The other prominent feature of the time-evolution conducted in the present study is the energy concentration around the lines of $l= \pm \sqrt{3} k$ in the cases of $\gamma \gg 1$. This energy concentration has also been reported by Connaughton et al. ${ }^{11}$ and Nazarenko, ${ }^{12}$ who explained this energy concentration as the energy diffusion in the two-dimensional wavenumber space along a contour of the coefficient that appears in the definition of zonostrophy. However, they dealt with cases involving localized initial energy distributions and finite values of $L_{\mathrm{D}} / L$, as stated above, and it is unclear whether their explanation holds for the cases considered in the present study, in which the two-dimensional energy spectra have broader distributions due to the initial isotropic spectrum, and the coefficient $\phi_{\mathbf{k}}$, which is defined by (24) in the long-wave limit, has a flat distribution in the wedge-shaped region. We believe that it may be possible to provide another explanation for the energy concentration based on the concept of near-resonant triad interactions, ${ }_{1}{ }^{14}$ and this concept may also provide an explanation for the region of low energy density near the $l$-axis, as shown in Figs. 3(c)-3(f). We would like to explore this possibility in the future.

Since the quasi-geostrophic equation arises from the modeling of geophysical fluids, it is natural to seek a counterpart of the energy accumulation shown above in the real atmosphere and ocean. In the atmosphere, however, the Rossby radius of deformation is on the order of thousands of kilometers, even for baroclinic modes, and it is difficult to find a regime in which the long-wave limit considered in the present paper is relevant. On the other hand, the Rossby radius of deformation for the first baroclinic Rossby waves in the middle-latitude ocean are several tens of kilometers, and it is possible for the long-wave limit to be relevant if the wavelength of the waves is sufficiently long. The characteristics of long baroclinic Rossby waves in the ocean have been clarified using data from satellite-borne altimeters. ${ }^{15}$ A recent spectral analysis by Glazman and Weichman ${ }^{16}$ revealed that the typical wavelength of the baroclinic Rossby waves is from several hundreds to one thousand kilometers, which is more than ten times as large as the Rossby radius of deformation. They also showed that "typical directions of characteristic wavenumber vector deviate from the zonal by $50^{\circ}-$ $80^{\circ}$." Here, "zonal" refers to the direction of $k$-axis in the two-dimensional wavenumber space. This deviation resembles the angular distribution of energy for the $\gamma \geq 1$ cases shown in Fig. 4 in the present paper, and the anisotropy of the energy cascade in the long-wave limit may be the cause of the observed deviation. This possibility was suggested by Balk and Zakharov ${ }^{9}$ and Balk. ${ }^{10}$ As stated above, however, in order for the anisotropy to be significant, the parameter $\gamma=\beta L_{\mathrm{D}}^{2} / U$ should be larger than unity, and whether this condition is satisfied in the real ocean should be investigated. In 
Table I of Glazman and Weichman, ${ }^{16}$ the value of $\beta L_{\mathrm{D}}^{2}$ was estimated to be $5.8 \mathrm{~cm} / \mathrm{s}$ for $25^{\circ} \mathrm{N}$ latitude. If we estimate the typical velocity scale associated with the Rossby waves to be $U \sim 1 \mathrm{~cm} / \mathrm{s}$ based on the fact that the corresponding anomaly of the sea level is several $\mathrm{cm},{ }^{15}$ the value of $\gamma$ is estimated to be roughly $\gamma \sim 5$. Then, the condition is satisfied, and the conservation of zonostrophy may contribute to the observed deviation. Confirming the condition for $\gamma$ is not sufficient to validate the connection between the observed deviation and the conservation of zonostrophy because the system considered herein is highly idealized (two-dimensional flow, doubly-periodic boundary condition, no forcing term, etc.). We believe that further numerical/observational research should be performed in order to examine this connection.

\section{ACKNOWLEDGMENTS}

The authors thank two anonymous reviewers for their helpful and constructive comments on this work. The ISPACK numerical library (http://www.gfd-dennou.org/arch/ispack/) was used for the computations, and the GFD-DENNOU Library (http://www.gfd-dennou.org/arch/dcl/) was used to draw the figures. Izumi Saito is supported by the JSPS Research Fellowships.

\section{APPENDIX A: DERIVATION OF THE LONG-WAVE LIMIT OF ZONOSTROPHY}

In this appendix, we derive an appropriate asymptotic form of zonostrophy for the long-wave limit. Here, in order to simplify the limiting process, we adopt a different nondimensionalization from that of the main text as

$$
x=L_{\mathrm{D}} x_{*}, \quad y=L_{\mathrm{D}} y_{*},
$$

rather than (8). Letting $(k, l)$ be the wavenumbers corresponding to this nondimensionalized coordinate, the original function for zonostrophy discovered by Balk ${ }^{6}$ is written as follows:

$$
\Phi_{\mathbf{k}}=\log \left(\frac{l+\sqrt{3} k+\mathrm{i}|\mathbf{k}|^{2}}{l-\sqrt{3} k+\mathrm{i}|\mathbf{k}|^{2}}\right) .
$$

Taking the imaginary part of the principal value (the argument is in the range of $(-\pi, \pi])$ ) of (A2) yields (21). We examine the long-wave limit $(|\mathbf{k}| \rightarrow 0)$ of (A2) separately for each region in the two-dimensional wavenumber space as follows:

- In the region in which $k>0$ and $|l|<\sqrt{3} k$.

We consider the limit of $|\mathbf{k}| \rightarrow 0$ along a line, $l=h k$, where $-\sqrt{3}<h<\sqrt{3}$ and $k>0$. Considering that $l+\sqrt{3} k>0$ and $l-\sqrt{3} k<0$ in this region, $\Phi_{\mathbf{k}}$ is expanded as follows:

$$
\begin{aligned}
\Phi_{\mathbf{k}} & =\log \left(\frac{l+\sqrt{3} k}{l-\sqrt{3} k}\right)+\log \left(1+\frac{\mathrm{i}|\mathbf{k}|^{2}}{l+\sqrt{3} k}\right)-\log \left(1+\frac{\mathrm{i}|\mathbf{k}|^{2}}{l-\sqrt{3} k}\right) \\
& =\log \left(\frac{l+\sqrt{3} k}{-(l-\sqrt{3} k)}\right)+\log (-1)+\left(\frac{\mathrm{i}|\mathbf{k}|^{2}}{l+\sqrt{3} k}-\frac{\mathrm{i}|\mathbf{k}|^{2}}{l-\sqrt{3} k}\right)+O\left(|\mathbf{k}|^{2}\right) \\
& =\log \left|\frac{l+\sqrt{3} k}{l-\sqrt{3} k}\right|+\mathrm{i}(\pi+2 n \pi)+\mathrm{i}\left(-2 \sqrt{3} \cdot \frac{k|\mathbf{k}|^{2}}{l^{2}-3 k^{2}}\right)+O\left(|\mathbf{k}|^{2}\right) .
\end{aligned}
$$

Here, Log indicates the principal value of the logarithm, and $n$ is an arbitrary integer, which comes from the multivaluedness of the logarithm. Since the value in the second set of parentheses in the last line of (A3) is positive (but infinitesimally small), we should choose $n=-1$ in order to obtain 
the principal value of $\Phi_{\mathbf{k}}$. Then, in this region, we obtain

$$
\begin{aligned}
\operatorname{Im}\left(\operatorname{pv}\left(\Phi_{\mathbf{k}}\right)\right) & =-\pi-2 \sqrt{3} \cdot \frac{k|\mathbf{k}|^{2}}{l^{2}-3 k^{2}}+O\left(|\mathbf{k}|^{2}\right) \\
& =-\pi-2 \sqrt{3} k-8 \sqrt{3} \cdot \frac{k^{3}}{l^{2}-3 k^{2}}+O\left(|\mathbf{k}|^{2}\right)
\end{aligned}
$$

Here, $\operatorname{Im}(\cdot)$ is the imaginary part of $(\cdot)$, and $\operatorname{pv}(\cdot)$ is the principal value of $(\cdot)$.

- In the region in which $l>0$ and $l>\sqrt{3}|k|$ :

We consider the limit of $|\mathbf{k}| \rightarrow 0$ along a line, $k=h l$, where $-1 / \sqrt{3}<h<1 / \sqrt{3}$ and $l>0$. Considering that $l-\sqrt{3} k>0$ and $l+\sqrt{3} k>0$ in this region, $\Phi_{\mathbf{k}}$ is expanded as follows:

$$
\begin{aligned}
\Phi_{\mathbf{k}} & =\log \left(\frac{l+\sqrt{3} k}{l-\sqrt{3} k}\right)+\log \left(1+\frac{\mathrm{i}|\mathbf{k}|^{2}}{l+\sqrt{3} k}\right)-\log \left(1+\frac{\mathrm{i}|\mathbf{k}|^{2}}{l-\sqrt{3} k}\right) \\
& =\log \left(\frac{l+\sqrt{3} k}{l-\sqrt{3} k}\right)+\left(\frac{\mathrm{i}|\mathbf{k}|^{2}}{l+\sqrt{3} k}-\frac{\mathrm{i}|\mathbf{k}|^{2}}{l-\sqrt{3} k}\right)+O\left(|\mathbf{k}|^{2}\right) \\
& =\log \left|\frac{l+\sqrt{3} k}{l-\sqrt{3} k}\right|+\mathrm{i}(2 n \pi)+\mathrm{i}\left(-2 \sqrt{3} \cdot \frac{k|\mathbf{k}|^{2}}{l^{2}-3 k^{2}}\right)+O\left(|\mathbf{k}|^{2}\right) .
\end{aligned}
$$

Since the value in the second set of parentheses in the last line of (A5) is infinitesimally small, we should choose $n=0$ in order to obtain the principal value of $\Phi_{\mathbf{k}}$. Then, in this region, we obtain

$$
\operatorname{Im}\left(\operatorname{pv}\left(\Phi_{\mathbf{k}}\right)\right)=-2 \sqrt{3} k-8 \sqrt{3} \cdot \frac{k^{3}}{l^{2}-3 k^{2}}+O\left(|\mathbf{k}|^{2}\right)
$$

- On the line $l=\sqrt{3} k(k>0)$ :

Considering that $l-\sqrt{3} k=0$ and $l+\sqrt{3} k>0$ on this line, $\Phi_{\mathbf{k}}$ is expanded as follows:

$$
\begin{aligned}
\Phi_{\mathbf{k}} & =\log \left(\frac{l+\sqrt{3} k+\mathrm{i}|\mathbf{k}|^{2}}{\mathrm{i}|\mathbf{k}|^{2}}\right) \\
& =\log \left(\frac{l+\sqrt{3} k}{|\mathbf{k}|^{2}}\right)-\log (\mathrm{i})+\log \left(1+\frac{\mathrm{i}|\mathbf{k}|^{2}}{l+\sqrt{3} k}\right) \\
& =\log \left|\frac{l+\sqrt{3} k}{|\mathbf{k}|^{2}}\right|-\mathrm{i} \frac{\pi}{2}+\mathrm{i}(2 n \pi)+\mathrm{i}\left(\frac{|\mathbf{k}|^{2}}{l+\sqrt{3} k}\right)+O\left(|\mathbf{k}|^{2}\right) .
\end{aligned}
$$

Since the value in the second set of parentheses in the last line of (A7) is infinitesimally small, we should choose $n=0$ in order to obtain the principal value of $\Phi_{\mathbf{k}}$. Then, on the line $l=\sqrt{3} k(k>0)$, we obtain

$$
\operatorname{Im}\left(\operatorname{pv}\left(\Phi_{\mathbf{k}}\right)\right)=-\frac{\pi}{2}-2 \sqrt{3} k+8 \sqrt{3} \cdot \frac{k}{3}+O\left(|\mathbf{k}|^{2}\right)
$$


Applying similar procedures to those described above to the other regions and lines and omitting $O\left(|\mathbf{k}|^{2}\right)$ terms, we finally obtain

$$
\operatorname{Im}\left(\operatorname{pv}\left(\Phi_{\mathbf{k}}\right)\right)=\left\{\begin{array}{lll}
0 & -2 \sqrt{3} k-8 \sqrt{3}\left(\frac{k^{3}}{l^{2}-3 k^{2}}\right) & (|l|>\sqrt{3}|k|) \\
-\pi & -2 \sqrt{3} k-8 \sqrt{3}\left(\frac{k^{3}}{l^{2}-3 k^{2}}\right) & (|l|<\sqrt{3}|k| \text { and } k>0) \\
\pi & -2 \sqrt{3} k-8 \sqrt{3}\left(\frac{k^{3}}{l^{2}-3 k^{2}}\right) & (|l|<\sqrt{3}|k| \text { and } k<0) \\
-\frac{\pi}{2} & -2 \sqrt{3} k-8 \sqrt{3}\left(-\frac{k}{3}\right) & (l= \pm \sqrt{3} k \text { and } k>0) \\
\frac{\pi}{2} & -2 \sqrt{3} k-8 \sqrt{3}\left(-\frac{k}{3}\right) & (l= \pm \sqrt{3} k \text { and } k<0)
\end{array} .\right.
$$

If we define $\eta_{\mathbf{k}}=-\operatorname{Im}\left(\operatorname{pv}\left(\Phi_{\mathbf{k}}\right)\right) / \pi$ and omit $O\left(|\mathbf{k}|^{1}\right)$ terms, we obtain (23). In (23), we choose $\eta_{(0,0)}$ $=0$ in order to satisfy (19). Note that the form of (22) appears as an $O\left(|\mathbf{k}|^{1}\right)$ term in (A9).

\section{APPENDIX B: PROOF THAT THE $\eta_{k}$ SATISFIES THE CONSTRAINT}

In this appendix, we present a proof that the $\eta_{\mathbf{k}}$ defined by (23) satisfies the constraint (19) whenever the condition (20) holds. The condition (20) can be rewritten as follows:

$$
\begin{aligned}
k_{0}+k_{1}+k_{2} & =0, \\
l_{0}+l_{1}+l_{2} & =0, \\
k_{0}\left(k_{0}^{2}+l_{0}^{2}\right)+k_{1}\left(k_{1}^{2}+l_{1}^{2}\right)+k_{2}\left(k_{2}^{2}+l_{2}^{2}\right) & =0 .
\end{aligned}
$$

First, let us consider the case of $\left|\mathbf{k}_{0}\right|=0$, namely, $\mathbf{k}_{0}=(0,0)$. In this case, the condition (B1) is satisfied when $\mathbf{k}_{2}=-\mathbf{k}_{1}$. For this choice of $\mathbf{k}_{0}, \mathbf{k}_{1}$, and $\mathbf{k}_{2}$, the constraint (19) is satisfied since $\eta_{(0,0)}=0$ and $\eta_{\mathbf{k}}=-\eta_{-\mathbf{k}}$ by the definition of (23).

Next, let us consider the case in which $\left|\mathbf{k}_{0}\right| \neq 0$. Since whether condition (B1) holds and whether constraint (19) is satisfied depend on neither the scale transform: $\left(\mathbf{k}_{0}, \mathbf{k}_{1}, \mathbf{k}_{2}\right) \mapsto\left(r \mathbf{k}_{0}, r \mathbf{k}_{1}, r \mathbf{k}_{2}\right)(r$ $\neq 0)$ nor the reflection transforms: $\left(\left(k_{0}, l_{0}\right),\left(k_{1}, l_{1}\right),\left(k_{2}, l_{2}\right)\right) \mapsto\left(\left(-k_{0}, l_{0}\right),\left(-k_{1}, l_{1}\right),\left(-k_{2}, l_{2}\right)\right)$ and $\left(\left(k_{0}, l_{0}\right),\left(k_{1}, l_{1}\right),\left(k_{2}, l_{2}\right)\right) \mapsto\left(\left(k_{0},-l_{0}\right),\left(k_{1},-l_{1}\right),\left(k_{2},-l_{2}\right)\right)$, we can restrict our attention to the case in which $\left(k_{0}, l_{0}\right)=\left(\cos \theta_{0}, \sin \theta_{0}\right)\left(0 \leq \theta_{0} \leq \pi / 2\right)$, without loss of generality. Eliminating $k_{1}$ and $l_{1}$ and using $k_{0}^{2}+l_{0}^{2}=1$ in (B1) yields

$$
\left(3 k_{0}\right) k_{2}^{2}+\left(2 l_{0}\right) k_{2} l_{2}+\left(k_{0}\right) l_{2}^{2}+\left(2 k_{0}^{2}+1\right) k_{2}+\left(2 k_{0} l_{0}\right) l_{2}=0 .
$$

This is the equation of a conic section in the two-dimensional wavenumber space for $\left(k_{2}, l_{2}\right)$. Since the discriminant is written as

$$
\begin{aligned}
\left(l_{0}\right)^{2}-\left(3 k_{0}\right)\left(k_{0}\right) & =\sin ^{2} \theta_{0}-3 \cos ^{2} \theta_{0} \\
& =4 \sin \left(\theta_{0}+\frac{\pi}{3}\right) \sin \left(\theta_{0}-\frac{\pi}{3}\right),
\end{aligned}
$$

the locus of $\mathbf{k}_{2}=\left(k_{2}, l_{2}\right)$ is either an ellipse (when $0 \leq \theta_{0}<\pi / 3$ ), or two hyperbolas (when $\pi / 3$ $<\theta_{0} \leq \pi / 2$ ), or two lines (when $\theta_{0}=\pi / 3$ ). This geometric property of condition (B1) was noted by Longuet-Higgins and Gill. ${ }^{17}$ Since Eq. (B2) holds, by replacing $\left(k_{2}, l_{2}\right)$ with $\left(k_{1}, l_{1}\right)$, the locus of $\mathbf{k}_{1}$ is either an ellipse, two hyperbolas, or two lines. The two points $\mathbf{k}_{1}$ and $\mathbf{k}_{2}$ are symmetric with respect to the point $-\mathbf{k}_{0} / 2$ in order to satisfy $\mathbf{k}_{0}+\mathbf{k}_{1}+\mathbf{k}_{2}=\mathbf{0}$. In the following, we present a geometric proof of the proposition. For convenience, we define five sets corresponding to the value 
of $\eta_{\mathbf{k}}$ as follows:

$$
\begin{array}{ll}
W_{0}=\{(k, l)|| l|>\sqrt{3}| k \mid \text { or } k=l=0\} & : \eta_{\mathbf{k} \in W_{0}}=0, \\
U_{+}=\{(k, l)|| l \mid<\sqrt{3} k\} & : \eta_{\mathbf{k} \in U_{+}}=1, \\
U_{-}=\{(k, l)|-| l \mid>\sqrt{3} k\} & : \eta_{\mathbf{k} \in U_{-}}=-1, \\
H_{+}=\{(k, l)|| l \mid=\sqrt{3} k \text { and } k>0\} & : \eta_{\mathbf{k} \in H_{+}}=1 / 2, \\
H_{-}=\{(k, l)|| l \mid=-\sqrt{3} k \text { and } k<0\} & : \eta_{\mathbf{k} \in H_{-}}=-1 / 2,
\end{array}
$$

and two points depending on the coordinates of $\mathbf{k}_{0}$ as follows:

$$
\begin{aligned}
& \mathbf{k}_{\mathrm{A}}=\left(-\frac{\sqrt{3} k_{0}+l_{0}}{2 \sqrt{3}},-\frac{\sqrt{3} k_{0}+l_{0}}{2}\right), \\
& \mathbf{k}_{\mathrm{B}}=\left(-\frac{\sqrt{3} k_{0}-l_{0}}{2 \sqrt{3}},-\frac{\sqrt{3} k_{0}-l_{0}}{2}\right) .
\end{aligned}
$$

Each set corresponds to a region in the wavenumber space, as shown in Fig. 8(a). Next, we present the proof for each of three ranges of $\theta_{0}$.

- $0 \leq \theta_{0}<\pi / 3$

For this range of $\theta_{0}$, the point $\mathbf{k}_{0} \in U_{+}$and the locus of $\mathbf{k}_{1}$ and $\mathbf{k}_{2}$ is an ellipse. Figure 8(b) shows the ellipse for the case in which $\theta_{0}=\pi / 6$ (for any value of $\theta_{0}$ in $[0, \pi / 3$ ), the following

(a)
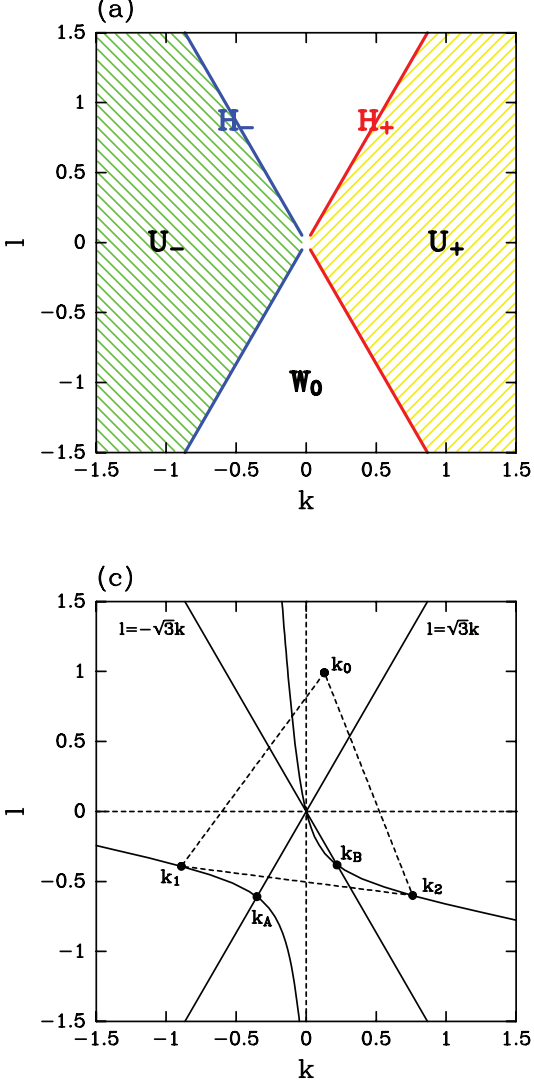

(b)
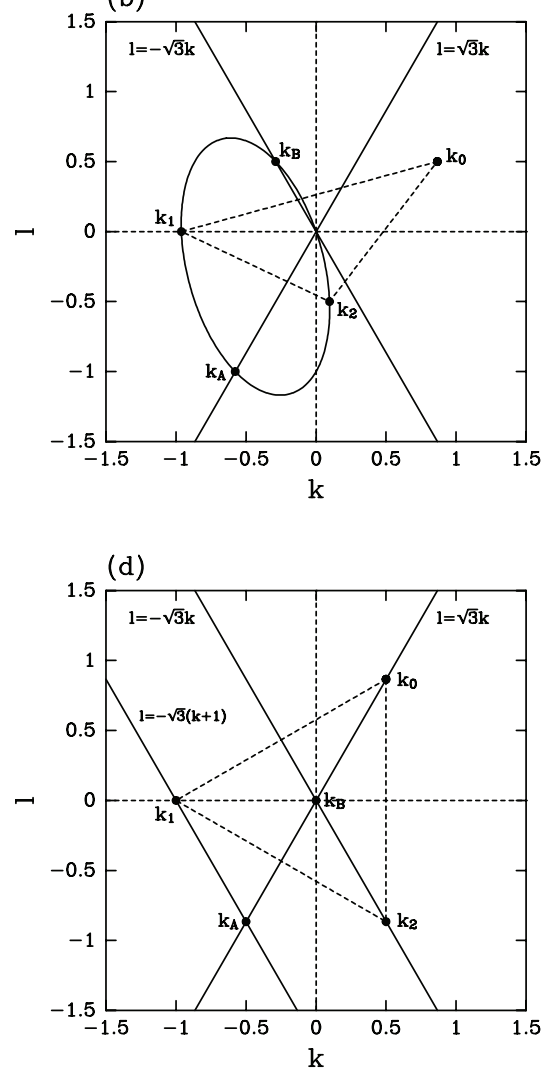

FIG. 8. (a) Regions in two-dimensional wavenumber space corresponding to the sets $W_{0}, U_{+}, U_{-}, H_{+}$, and $H_{-}$, which are defined by (B3). (b)-(d) The loci of $\mathbf{k}_{1}$ and $\mathbf{k}_{2}$ that satisfy (B1) when $\theta_{0}=\pi / 6$ (b), $\theta_{0}=11 \pi / 24$ (c), and $\theta_{0}=\pi / 3$ (d). See the text for details. 
explanation applies). The ellipse crosses the line $l=\sqrt{3} k$ at the origin and the point $\mathbf{k}_{\mathrm{A}}$ and crosses the line $l=-\sqrt{3} k$ at the origin and the point $\mathbf{k}_{\mathrm{B}}$. When the point $\mathbf{k}_{1}$ coincides with $\mathbf{k}_{\mathrm{A}}$, the point $\mathbf{k}_{2}$ coincides with $\mathbf{k}_{\mathrm{B}}$. Then, $\mathbf{k}_{1}, \mathbf{k}_{2} \in H_{-}$and (19) holds because $\eta_{\mathbf{k}_{1}}=\eta_{\mathbf{k}_{2}}=-1 / 2$ and $\eta_{\mathbf{k}_{0}}=1$ in this case. When the point $\mathbf{k}_{1}$ is on the arc of the ellipse that lies in the set $U_{-}$, the point $\mathbf{k}_{2}$ is on the arc of the ellipse that lies in the set $W_{0}$. Then, $\mathbf{k}_{1} \in U_{-}$and $\mathbf{k}_{2} \in W_{0}$ and (19) holds because $\eta_{\mathbf{k}_{1}}=-1, \eta_{\mathbf{k}_{2}}=0$, and $\eta_{\mathbf{k}_{0}}=1$ in this case. The above explanation holds even if $\mathbf{k}_{1}$ is exchanged with $\mathbf{k}_{2}$. Therefore, the proof is complete for this range of $\theta_{0}$.

- $\pi / 3<\theta_{0} \leq \pi / 2$

For this range of $\theta_{0}$, the point $\mathbf{k}_{0} \in W_{0}$ and the locus of $\mathbf{k}_{1}$ and $\mathbf{k}_{2}$ is given by two hyperbolas. Figure 8(c) shows the hyperbolas for the case of $\theta_{0}=11 \pi / 24$ (for any value of $\theta_{0}$ in $(\pi / 3, \pi / 2$ ], the following explanation applies). The hyperbola at the lower left crosses the line $l=\sqrt{3} k$ at the point $\mathbf{k}_{\mathrm{A}}$, whereas the hyperbola at the upper right crosses the line $l=-\sqrt{3} k$ at the origin and the point $\mathbf{k}_{\mathrm{B}}$. When the point $\mathbf{k}_{1}$ coincides with $\mathbf{k}_{\mathrm{A}}$, the point $\mathbf{k}_{2}$ coincides with $\mathbf{k}_{\mathrm{B}}$. Then, $\mathbf{k}_{1} \in H_{-}$ and $\mathbf{k}_{2} \in H_{+}$so that (19) holds because $\eta_{\mathbf{k}_{1}}=-1 / 2, \eta_{\mathbf{k}_{2}}=1 / 2$ and $\eta_{\mathbf{k}_{0}}=0$ in this case. When the point $\mathbf{k}_{1}$ is on the arc of the lower left hyperbola that lies in the set $U_{-}$, the point $\mathbf{k}_{2}$ is on the arc of the upper right hyperbola that lies in the set $U_{+}$. Then, $\mathbf{k}_{1} \in U_{-}$and $\mathbf{k}_{2} \in U_{+}$so that (19) holds because $\eta_{\mathbf{k}_{1}}=-1, \eta_{\mathbf{k}_{2}}=1$, and $\eta_{\mathbf{k}_{0}}=0$ in this case. When the point $\mathbf{k}_{1}$ is on the arc of the lower left hyperbola that lies in the set $W_{0}$, the point $\mathbf{k}_{2}$ is on the arc of the upper right hyperbola that lies in the set $W_{0}$. Then, $\mathbf{k}_{1}, \mathbf{k}_{2} \in W_{0}$, and (19) holds because $\eta_{\mathbf{k}_{1}}=\eta_{\mathbf{k}_{2}}=0$, and $\eta_{\mathbf{k}_{0}}=0$ in this case. The above explanation holds even if $\mathbf{k}_{1}$ is exchanged with $\mathbf{k}_{2}$. Therefore, the proof is completed for this range of $\theta_{0}$.

- $\theta_{0}=\pi / 3$

For this value of $\theta_{0}$, the point $\mathbf{k}_{0} \in H_{+}$and the locus of $\mathbf{k}_{1}$ and $\mathbf{k}_{2}$ is given by the two lines, $l=-\sqrt{3} k$ and $l=-\sqrt{3}(k+1)$. Figure $8(\mathrm{~d})$ shows the two lines. Line $l=-\sqrt{3}(k+1)$ crosses line $l=\sqrt{3} k$ at point $\mathbf{k}_{\mathrm{A}}$, whereas line $l=-\sqrt{3} k$ crosses line $l=\sqrt{3} k$ at point $\mathbf{k}_{\mathrm{B}}$, which coincides with the origin. When the point $\mathbf{k}_{1}$ coincides with $\mathbf{k}_{\mathrm{A}}$, the point $\mathbf{k}_{2}$ coincides with $\mathbf{k}_{\mathrm{B}}$. Then, $\mathbf{k}_{1} \in H_{-}$ and $\mathbf{k}_{2} \in W_{0}$ so that (19) holds because $\eta_{\mathbf{k}_{1}}=-1 / 2, \eta_{\mathbf{k}_{2}}=0$, and $\eta_{\mathbf{k}_{0}}=1 / 2$ in this case. When the point $\mathbf{k}_{1}$ is on the ray of line $l=-\sqrt{3}(k+1)$ that lies in the set $U_{-}$, the point $\mathbf{k}_{2}$ is on the ray of line $l=-\sqrt{3} k$ that lies in the set $H_{+}$. Then, $\mathbf{k}_{1} \in U_{-}$and $\mathbf{k}_{2} \in H_{+}$so that (19) holds because $\eta_{\mathbf{k}_{1}}=-1$, $\eta_{\mathbf{k}_{2}}=1 / 2$, and $\eta_{\mathbf{k}_{0}}=1 / 2$ in this case. When the point $\mathbf{k}_{1}$ is on the ray of the line $l=-\sqrt{3}(k+1)$ that lies in the set $W_{0}$, the point $\mathbf{k}_{2}$ is on the ray of line $l=-\sqrt{3} k$ that lies in the set $H_{-}$. Then, $\mathbf{k}_{1} \in W_{0}$ and $\mathbf{k}_{2} \in H_{-}$so that (19) holds because $\eta_{\mathbf{k}_{1}}=0, \eta_{\mathbf{k}_{2}}=-1 / 2$, and $\eta_{\mathbf{k}_{0}}=1 / 2$ in this case. The above explanation holds even if $\mathbf{k}_{1}$ is exchanged with $\mathbf{k}_{2}$. Thus, the proof is completed for this value of $\theta_{0}$.

${ }^{1}$ P. B. Rhines, "Waves and turbulence on a beta-plane," J. Fluid Mech. 69, 417 (1975).

${ }^{2}$ G. K. Vallis and M. E. Maltrud, "Generation of mean flows and jets on a beta plane and over topography," J. Phys. Oceanogr. 23, 1346 (1993).

${ }^{3}$ A. Okuno and A. Masuda, "Effect of horizontal divergence on the geostrophic turbulence on a beta-plane: Suppression of the Rhines effect," Phys. Fluids 15, 56 (2003).

${ }^{4}$ V. D. Larichev and J. C. McWilliams, "Weakly decaying turbulence in an equivalent-barotropic fluid," Phys. Fluids A 3, 938 (1991).

${ }^{5}$ A. M. Balk, S. V. Nazarenko, and V. E. Zakharov, "A new invariant for drift turbulence," Phys. Lett. A 152, 276 (1991).

${ }^{6}$ A. M. Balk, “A new invariant for Rossby wave systems,” Phys. Lett. A 155, 20 (1991).

${ }^{7}$ S. Nazarenko and B. Quinn, "Triple cascade behavior in quasigeostrophic and drift turbulence and generation of zonal jets," Phys. Rev. Lett. 103, 118501 (2009).

${ }^{8}$ A. M. Balk, "Angular distribution of Rossby wave energy," Phys. Lett. A 345, 154 (2005).

${ }^{9}$ A. M. Balk and V. E. Zakharov, "Cascade generation of zonal flows by the drift wave turbulence," Phys. Lett. A 373, 4049 (2009).

${ }^{10}$ A. M. Balk, F. van Heerden, and P. B. Weichman, "Rotating shallow water dynamics: Extra invariant and the formation of zonal jets," Phys. Rev. E 83, 046320 (2011).

${ }^{11}$ C. Connaughton, S. Nazarenko, and B. Quinn, "Feedback of zonal flows on wave turbulence driven by small-scale instability in the Charney-Hasegawa-Mima model,” Europhys. Lett. 96, 25001 (2011).

${ }^{12}$ S. Nazarenko, Wave Turbulence, Lecture Notes in Physics Vol. 825 (Springer, Berlin, 2011), p. 205. 
${ }^{13}$ A. M. Balk and F. van Heerden, "Conservation style of the extra invariant for Rossby waves," Physica D 223, 109 (2006).

${ }^{14}$ Y. Lee and L. M. Smith, "On the formation of geophysical and planetary zonal flows by near-resonant wave interactions," J. Fluid Mech. 576, 405 (2007).

${ }^{15}$ D. B. Chelton and M. G. Schlax, "Global observations of oceanic Rossby waves," Science 272, 234 (1996).

${ }^{16}$ R. E. Glazman and P. B. Weichman, "Meridional component of oceanic Rossby wave propagation," Dyn. Atmos. Oceans 38, 173 (2005)

${ }^{17}$ M. S. Longuet-Higgins and A. Gill, "Resonant interactions between planetary waves," Proc. R. Soc. London, Ser. A 299, 120 (1967). 